LA-UR-95-1648

\title{
Extracting source parameters from gaussian fits to two-particle correlations
}

\author{
Scott Chapman and J. Rayford Nix \\ Theoretical Division, Los Alamos National Laboratory, Los Alamos, NM 87545, USA \\ Ulrich Heinz \\ Institut für Theoretische Physik, Universität Regensburg, \\ D-93040 Regensburg, Germany
}

(August 9, 1995)

\begin{abstract}
Using a quadratic saddle-point approximation, we show how information about a particle-emitting source can be extracted from gaussian fits to twoparticle correlation data. Although the formalism is completely general, extraction of the relevant parameters is much simpler for sources within an interesting class of azimuthally symmetric models. After discussing the standard fitting procedure, we introduce a new gaussian fitting procedure which is an azimuthally symmetric generalization of the Yano-Koonin formalism for spherically symmetric sources. This new fitting procedure has the advantage that in addition to being able to measure source parameters in a fixed frame or the longitudinally co-moving system, it can also measure these parameters in the local rest frame of the source.
\end{abstract}

PACS: 25.70.Pq 


\section{INTRODUCTION}

Recently quite a bit of work has been done in trying to determine which attributes of the hadronic source formed in high-energy particle or heavy-ion collisions can be determined by measuring the Hanbury-Brown-Twiss (HBT) correlations of identical emitted particles. Usually the experimental correlation function is fit with a gaussian in some components of the four-momentum difference $q=p_{1}-p_{2}$ [1 [5]. The parameters of such a fit (called correlation radii) are then often compared to some simple analytic model in order to get an idea of what is being measured. For example, for a static gaussian source, the duration of emission time is directly proportional to the difference of the squares of the correlation radii which are parallel to ("out") and perpendicular to ("side") the transverse component of the total pair momentum. However, this result is not true if for example the actual source contains any $z-t, z-x$, or $x$ - $t$ correlations, such as would be caused for quickly expanding sources.

The purpose of this paper is to determine exactly what features of the source are actually being measured by experimental correlation data. To do this, we use a quadratic saddlepoint approximation [6 8] to a general source function in order to derive a completely Lorentz covariant expression for the two-particle correlation function which can be applied to a wide range of analytic models. In this approximation, $10 \mathrm{~K}$-dependent parameters are needed

to describe a general source, where $\mathbf{K}=\frac{1}{2}\left(\mathbf{p}_{1}+\mathbf{p}_{2}\right)$ is the average momentum of the two particles. The origin of these 10 parameters can be understood by noting that for each value of $\mathbf{K}$, our approximation is mathematically equivalent to a gaussian ellipsoid described by three Euler angles of orientation, three components of the velocity of the local rest frame, three spatial extensions, and one temporal extension. As we will show, however, only six $\mathbf{K}$-dependent parameters (not including the chaoticity parameter $\lambda$ ) can be measured by making a gaussian fit in $q$ to the correlation function.

Furthermore, a source function which is azimuthally symmetric in coordinate space will not in general be azimuthally symmetric in momentum space, since the direction defined by 
$K_{\perp}$ breaks this symmetry. As a result, the $\mathbf{K}$-dependent ellipsoid equivalent to such a source still requires one Euler angle, two velocity components, three spatial extensions, and one temporal extension in order to describe it. In this case, only four $\mathbf{K}$-dependent parameters can be determined by making a gaussian fit in $q$. Since for arbitrary $K_{\perp}$ the number of source parameters exceeds the number of gaussian-fit parameters, some definite model must be used in order to interpret how the latter depend on the former.

For pairs with $K_{\perp}=0$, however, only four $\mathbf{K}$-dependent parameters are needed to describe the source (one velocity component, two spatial extensions and one temporal extension), so the four parameters measured in a gaussian fit provide enough information to unambiguously determine all of these source parameters. Although in practice it is very difficult to measure a pair whose total transverse momentum vanishes, there is an interesting class of models in which the $K_{\perp}=0$ simplifications persist for nonzero values of $K_{\perp}$. In Sec. 5 we study a hydrodynamical model similar to ones which have been used to fit one-particle distributions from heavy-ion collisions at the Brookhaven Alternating Gradient Synchrotron (AGS) and the CERN Super Proton Synchrotron (SPS). Within the context of this model, one would expect the simplifications in the extraction of source parameters to occur for pairs whose average energy in the measurement frame is less than the freezeout temperature divided by the square of the transverse flow velocity (about $560 \mathrm{MeV}$ for $\mathrm{Si}+\mathrm{Au}$ at the AGS or $\mathrm{S}+\mathrm{Pb}$ at the $\mathrm{SPS})$.

If the correlation function for a model in the afore-mentioned class is fit to a gaussian in the spatial components of $q$, then in order to extract the relevant source parameters it is crucial to pick beforehand the correct longitudinal reference frame for the measurement. If, on the other hand, a fit is made to a generalization of the Yano-Koonin formalism [9.10], the correlation radii will automatically measure the relevant source parameters even if the wrong frame is chosen for measurement. For example, for a finite longitudinally expanding source, the new formalism allows measurement of the source parameters in the local rest frame of the fluid, whereas fixed and longitudinally co-moving system (LCMS) radii measure the source in different frames. 


\section{LORENTZ COVARIANT CORRELATION FUNCTION}

For the two-particle correlation function, we use the well-established theoretical approximation 8, 11, 12,

$$
C(\mathbf{q}, \mathbf{K}) \simeq 1 \pm \frac{\left|\int d^{4} x S(x, K) e^{i q \cdot x}\right|^{2}}{\left|\int d^{4} x S(x, K)\right|^{2}}
$$

where $\mathbf{q}=\mathbf{p}_{1}-\mathbf{p}_{\mathbf{2}}, q_{0}=E_{1}-E_{2}, \mathbf{K}=\frac{1}{2}\left(\mathbf{p}_{1}+\mathbf{p}_{2}\right)$, and $K_{0}=E_{K}=\sqrt{m^{2}+|\mathbf{K}|^{2}}$. The plus sign is to be used for boson pairs and the minus sign for fermion pairs. The labeling of particles 1 and 2 is defined such that $q_{1}=q_{x}$ is always positive. In this way, pairs with positive $q_{2}=q_{y}$ and/or $q_{3}=q_{z}$ are physically distinct from those with negative $q_{2}$ and/or $q_{3}$. The $S(x, K)$ in Eq. (2.1) is a function which describes the phase-space density of the emitting source.

The spacetime saddle point $\bar{x}(\mathbf{K})$ of the emission function $S(x, K)$ is defined via the four equations [6]

$$
\left.\frac{\partial}{\partial x_{\mu}} \ln S(x, K)\right|_{\bar{x}}=0
$$

where $\mu=\{0,1,2,3\}$. The saddle point is that point in spacetime which has the maximum probability of emitting a particle with momentum K. A quadratic saddle-point approximation for $S(x, K)$ then yields

$$
S(x, K) \simeq S(\bar{x}, K) \exp \left[-\frac{1}{2}(x-\bar{x})^{\mu}(x-\bar{x})^{\nu} B_{\mu \nu}(\mathbf{K})\right]
$$

where the symmetric curvature tensor $B_{\mu \nu}$ is given by

$$
B_{\mu \nu}(\mathbf{K})=-\left.\partial_{\mu} \partial_{\nu} \ln S(x, K)\right|_{\bar{x}}
$$

We define the curvature radius in the $\mu$ th direction by [6,7]

$$
\lambda_{\mu}(\mathbf{K})=\left[B_{\mu \mu}(\mathbf{K})\right]^{-1 / 2}
$$

Note that since $B_{\mu \nu}(\mathbf{K})$ is symmetric, it will in general have 10 independent components. From the form of Eq. (2.3) it is seen that the saddle-point approximation is mathematically 
equivalent to an ellipsoid described by the $10 \mathbf{K}$-dependent parameters mentioned in the Introduction. As long as the saddle point $\bar{x}(\mathbf{K})$ is unique, knowledge of the 10 functions $B_{\mu \nu}(\mathbf{K})$ is in most practical situations sufficient for a complete characterization of the source.

It is convenient to define [8,13,14] the following $\mathbf{K}$-dependent average of an arbitrary spacetime function $\xi(x)$ with the source density $S(x, K)$ :

$$
\langle\xi\rangle \equiv\langle\xi(x)\rangle(\mathbf{K})=\frac{\int d^{4} x \xi(x) S(x, K)}{\int d^{4} x S(x, K)} .
$$

Using this notation, the correlation function (2.1) can be compactly written as

$$
C(\mathbf{q}, \mathbf{K}) \simeq 1 \pm\left|\left\langle e^{i q \cdot x}\right\rangle(\mathbf{K})\right|^{2}
$$

Furthermore, within the saddle-point approximation (2.3), the following relations hold:

$$
\begin{gathered}
\left\langle x_{\mu}\right\rangle=\bar{x}_{\mu}(\mathbf{K}) \\
\left\langle x_{\mu} x_{\nu}\right\rangle-\left\langle x_{\mu}\right\rangle\left\langle x_{\nu}\right\rangle=\left(B^{-1}\right)_{\mu \nu}(\mathbf{K}) .
\end{gathered}
$$

The saddle point is thus the average spacetime point from which particle pairs with momentum $\mathbf{K}$ are emitted, and the components of the inverse of the curvature tensor $\left(B^{-1}\right)_{\mu \nu}(\mathbf{K})$ give the spacetime correlations of the source. The four diagonal elements

$$
\left(B^{-1}\right)_{\mu \mu}(\mathbf{K})=\left\langle x_{\mu}^{2}\right\rangle-\left\langle x_{\mu}\right\rangle^{2}
$$

can be understood as the squares of the lengths of homogeneity of the source as seen by pairs with momentum $\mathbf{K}$. It should be noted that the homogeneity lengths agree with the curvature radii (2.5) only if the curvature tensor $B_{\mu \nu}$ is diagonal. This was implicitly assumed by the authors of [6,7], who first introduced the name "homogeneity length" but used it for the curvature radii (2.5) of the source near the saddle point.

Within the approximations of Eqs. (2.1) and (2.3), calculation of a general correlation function is straightforward, yielding

$$
C(\mathbf{q}, \mathbf{K})=1 \pm \exp \left[-q^{\mu} q^{\nu}\left(B^{-1}\right)_{\mu \nu}\right]
$$


From Eq. (2.9) we can see that the correlation function directly measures the spacetime correlations within the source.

It may at first seem that all of the components of the correlation tensor $B^{-1}$ can be found simply by comparing the results of a four-dimensional fit to the correlation function with Eq. (2.11). Such a fit is not possible, however, since $q_{0}$ is highly correlated with the other components of $\mathbf{q}$ through the equation

$$
q_{0}=\sum_{i} \beta_{i} q_{i}, \quad \text { where } \quad \beta_{i}=\frac{2 K_{i}}{E_{1}+E_{2}} .
$$

By making the approximation

$$
\beta_{i} \simeq K_{i} / E_{K}
$$

which is valid for pairs with $|\mathbf{q}| \ll E_{K}$, one can use (2.12) to fit the correlation function (2.11) to the form

$$
\begin{aligned}
C(\mathbf{q}, \mathbf{K})=1 \pm \lambda \exp [ & -q_{1}^{2} R_{1}^{2}(\mathbf{K})-q_{2}^{2} R_{2}^{2}(\mathbf{K})-q_{3}^{2} R_{3}^{2}(\mathbf{K}) \\
& \left.-2 q_{1} q_{2} R_{12}^{2}(\mathbf{K})-2 q_{1} q_{3} R_{13}^{2}(\mathbf{K})-2 q_{2} q_{3} R_{23}^{2}(\mathbf{K})\right],
\end{aligned}
$$

where the $R_{i j}^{2}$ cross terms can be either positive or negative and $\lambda$ is a parameter introduced to allow for coherence effects [15, 16] and/or particles from the decay of long-lived resonances [17 20]. From (2.9) and (2.12) the six functions $R_{i j}^{2}(\mathbf{K})\left(R_{i} \equiv R_{i i}\right)$ can be expressed as the correlations [8,14]

$$
R_{i j}^{2}(\mathbf{K})=\left\langle\left(x_{i}-\beta_{i} t\right)\left(x_{j}-\beta_{j} t\right)\right\rangle-\left\langle x_{i}-\beta_{i} t\right\rangle\left\langle x_{j}-\beta_{j} t\right\rangle .
$$

In general, the six $R^{2}$ parameters found by fitting correlations to Eq. (2.14) do not provide enough information to determine the 10 independent components of $\left(B^{-1}\right)_{\mu \nu}$.

Due to Eqs. (2.12) and (2.13), any Lorentz transformation and/or spatial rotation to a new coordinate system can be written as a purely spatial linear transformation,

$$
q_{i}^{\prime}=a_{i j} q_{j}
$$


Thus the new $R^{\prime 2}$ parameters found in the primed frame will simply be linear combinations of the $R^{2}$ parameters found in the original frame. For example, the longitudinally co-moving system (LCMS) is defined as the longitudinally boosted frame in which $\beta_{3}^{\prime}=0$ [4,5, 21]. The $R^{\prime 2}$ parameters in this (primed) frame are related to those in some fixed (unprimed) frame via

$$
\begin{aligned}
& R_{1}^{\prime 2}=R_{1}^{2}+\gamma^{4} \beta_{1}^{2} \beta_{3}^{2} R_{3}^{2}+2 \gamma^{2} \beta_{1} \beta_{3} R_{13}^{2} \\
& R_{2}^{\prime 2}=R_{2}^{2}+\gamma^{4} \beta_{2}^{2} \beta_{3}^{2} R_{3}^{2}+2 \gamma^{2} \beta_{2} \beta_{3} R_{23}^{2} \\
& R_{3}^{\prime 2}=\gamma^{2} R_{3}^{2} \\
& R_{12}^{\prime 2}=R_{12}^{2}+\gamma^{2} \beta_{2} \beta_{3} R_{13}^{2}+\gamma^{2} \beta_{1} \beta_{3} R_{23}^{2}+\gamma^{4} \beta_{1} \beta_{2} \beta_{3}^{2} R_{3}^{2} \\
& R_{13}^{\prime 2}=\gamma R_{13}^{2}+\gamma^{3} \beta_{1} \beta_{3} R_{3}^{2} \\
& R_{23}^{\prime 2}=\gamma R_{23}^{2}+\gamma^{3} \beta_{2} \beta_{3} R_{3}^{2},
\end{aligned}
$$

where the $\beta_{i}$ are measured in the fixed frame and $\gamma=1 / \sqrt{1-\beta_{3}^{2}}$. The above equalities can be used as an experimental test of the validity of the saddle point approximation in the following way: If measured LCMS radii are not equal to the above corresponding combinations of fixed-frame radii, then the saddle point formalism is not a good approximation to the actual correlation function.

For sources which have highly non-gaussian spacetime dependencies, it is better to define [22] the inverse of the curvature tensor directly through Eqns. (2.9) and (2.6) (using the full source $S(x, K))$ rather than to use second derivatives to define the curvature tensor at the saddle point via (2.4). A simple example of when this is necessary is provided by the emission function from a uniform sphere:

$$
S(x, K)=f(K) \delta\left(t-t_{0}\right) \theta(R-r)
$$

where $r=\sqrt{x^{2}+y^{2}+z^{2}}$ and $R$ is the radius of the sphere. For this source function, the saddle point is not unique and second derivatives are ill-defined. Nevertheless, $\left(B^{-1}\right)_{\mu \nu}$ in (2.9) is perfectly well defined, producing through (2.11) a gaussian correlation function of the form 


$$
C(\mathbf{q}, \mathbf{K})=1 \pm \exp \left(-|\mathbf{q}|^{2} R^{2} / 5\right)
$$

The reader can verify that the above expression is a good approximation to the correlation function

$$
C(\mathbf{q}, \mathbf{K})=1 \pm 9\left[\frac{\cos (|\mathbf{q}| R)}{(|\mathbf{q}| R)^{2}}-\frac{\sin (|\mathbf{q}| R)}{(|\mathbf{q}| R)^{3}}\right]^{2},
$$

which is found by plugging (2.18) directly into (2.1). This example clearly shows that in terms of the correlation function, even a highly non-gaussian source can be well approximated by a gaussian with the same rms width.

\section{AZIMUTHALLY SYMMETRIC SOURCES}

For an azimuthally symmetric source, it is convenient to choose $\hat{z}$ to point along the beam ("longitudinal") axis and to choose $\hat{x}$ to point in the same direction as the component of $\mathbf{K}$ which is perpendicular to the beam ("out"). The remaining ("side") direction is then defined by $\hat{y}=\hat{z} \times \hat{x}$ [23]. Note that by definition $K_{1}$ and $\beta_{1}$ are always positive and $K_{2}=\beta_{2}=0$. Since the latter is true, azimuthally symmetric sources must satisfy

$$
S(t, x, y, z, K)=S(t, x,-y, z, K) .
$$

From Eq. (2.3) this implies that $\bar{y}=B_{\mu 2}=B_{2 \mu}=0$ for $\mu \neq 2$, so for azimuthally symmetric sources $B_{\mu \nu}$ has only seven independent components.

By inserting Eq. (3.1) into Eq. (2.1), one can see that the correlation function from an azimuthally symmetric source must be unchanged under the substitution $q_{2} \rightarrow-q_{2}$. This

implies that $R_{12}^{2}=R_{23}^{2}=0$, so that only four $R^{2}$ parameters can be found by making a gaussian fit to the correlation function [8, 14, 24]:

$$
C(\mathbf{q}, \mathbf{K})=1 \pm \lambda \exp \left[-q_{1}^{2} R_{1}^{2}(\mathbf{K})-q_{2}^{2} R_{2}^{2}(\mathbf{K})-q_{3}^{2} R_{3}^{2}(\mathbf{K})-2 q_{1} q_{3} R_{13}^{2}(\mathbf{K})\right]
$$

Expressing these four correlation radii in terms of the seven independent elements of the curvature tensor we have 


$$
\begin{aligned}
R_{2}^{2}= & \lambda_{2}^{2} \\
R_{1}^{2}=\Gamma & {\left[\lambda_{1}^{2}\left(1-\lambda_{0}^{2} \lambda_{3}^{2} B_{30}^{2}\right)+\beta_{1}^{2} \lambda_{0}^{2}\left(1-\lambda_{1}^{2} \lambda_{3}^{2} B_{31}^{2}\right)+2 \beta_{1} \lambda_{1}^{2} \lambda_{0}^{2}\left(B_{10}-\lambda_{3}^{2} B_{31} B_{30}\right)\right] } \\
R_{3}^{2}=\Gamma[ & \left.\lambda_{3}^{2}\left(1-\lambda_{0}^{2} \lambda_{1}^{2} B_{10}^{2}\right)+\beta_{3}^{2} \lambda_{0}^{2}\left(1-\lambda_{1}^{2} \lambda_{3}^{2} B_{31}^{2}\right)+2 \beta_{3} \lambda_{3}^{2} \lambda_{0}^{2}\left(B_{30}-\lambda_{1}^{2} B_{31} B_{10}\right)\right] \\
R_{13}^{2}=\Gamma[ & \beta_{1} \beta_{3} \lambda_{0}^{2}\left(1-\lambda_{1}^{2} \lambda_{3}^{2} B_{31}^{2}\right)+\beta_{1} \lambda_{3}^{2} \lambda_{0}^{2}\left(B_{30}-\lambda_{1}^{2} B_{31} B_{10}\right) \\
& \left.\quad+\beta_{3} \lambda_{1}^{2} \lambda_{0}^{2}\left(B_{10}-\lambda_{3}^{2} B_{31} B_{30}\right)-\lambda_{1}^{2} \lambda_{3}^{2}\left(B_{31}-\lambda_{0}^{2} B_{10} B_{30}\right)\right],
\end{aligned}
$$

where

$$
\Gamma=\left[1-\lambda_{0}^{2} \lambda_{1}^{2} B_{10}^{2}-\lambda_{1}^{2} \lambda_{3}^{2} B_{31}^{2}-\lambda_{0}^{2} \lambda_{3}^{2} B_{30}^{2}+2 \lambda_{0}^{2} \lambda_{1}^{2} \lambda_{3}^{2} B_{10} B_{30} B_{31}\right]^{-1}
$$

It is now apparent that the statement that $R_{1}^{2}-R_{2}^{2}$ is proportional to the square of the emission time is highly model dependent. Implicit in this statement are the assumptions that $\lambda_{1}=\lambda_{2}$ and that all $x-z, x-t$, and $z-t$ correlations are negligible.

Until now we have been discussing fits to the correlation function which are threedimensional gaussians in the spatial components of $q$. It is also possible to fit correlation functions in a different three-dimensional space defined by $q_{\perp}=\sqrt{q_{1}^{2}+q_{2}^{2}}, q_{3}$ and $q_{0}$. As we pointed out in the last section, $q_{0}$ is highly correlated with the spatial components of $q$ through Eq. (2.12) which we rewrite here as

$$
q_{0}=\beta_{1} q_{\perp} \cos \phi+\beta_{3} q_{3}
$$

where $\phi$ is the angle between $\hat{x}$ and $\mathbf{q}_{\perp}$. Due to this correlation, $q_{0}$ only varies over a finite range which is nonetheless greater than zero for any nonzero values of $\beta_{1}$ and $q_{\perp}$ :

$$
-\beta_{1} q_{\perp}+\beta_{3} q_{3} \leq q_{0} \leq \beta_{1} q_{\perp}+\beta_{3} q_{3} .
$$

Despite this phase-space limitation, it still possible to fit correlation functions with gaussians in a $\left(q_{\perp}, q_{3}, q_{0}\right)$ space [25].

For example, one could use an azimuthally symmetric generalization of the Yano-Koonin formalism [9,10]

$$
C(\mathbf{q}, \mathbf{K})=1 \pm \lambda \exp \left[-q_{\perp}^{2} R_{t}^{2}+\left(q_{0}^{2}-q_{3}^{2}\right) R_{4}^{2}-(q \cdot U)^{2}\left(R_{0}^{2}+R_{4}^{2}\right)\right],
$$


where

$$
U=\gamma(1,0,0, v), \quad \gamma=1 / \sqrt{1-v^{2}}
$$

(in units with $c=1$ ), and $R_{t}(\mathbf{K}), R_{0}(\mathbf{K}), R_{4}(\mathbf{K})$, and $v(\mathbf{K})$ are the four fit parameters. When using this fitting procedure, it is convenient to define the particle labeling such that $q_{0}$ is always positive. In this way, pairs with positive and negative $q_{3}$ are physically distinct and can be separately binned. For the remainder of this paper, we will refer to Eqs. (3.2) and (3.7) as the "standard" and "GYK" (generalized Yano-Koonin) fitting procedures, respectively.

The boost-invariant form of the "GYK" fit means that for any given value of $\mathbf{K}$ there is a longitudinally boosted reference frame in which $v(\mathbf{K})=0$. The fit parameters $R_{t}(\mathbf{K})$, $R_{0}(\mathbf{K})$, and $R_{4}(\mathbf{K})$ measure the source in this frame, regardless of which longitudinally boosted frame is chosen for the evaluation of the $q_{\mu}$. Although the general interpretation of the $v(\mathbf{K})=0$ frame is difficult, it can be shown that in this frame

$$
\left\langle\left(\beta_{1} z-\beta_{3} x\right)\left(\beta_{1} t-x\right)\right\rangle-\left\langle\beta_{1} z-\beta_{3} x\right\rangle\left\langle\beta_{1} t-x\right\rangle-\beta_{3}\left\langle y^{2}\right\rangle=0
$$

Furthermore, in this frame the remaining fit parameters take the form (for $\beta_{1} \neq 0$ )

$$
\begin{aligned}
& R_{t}^{2}=\left\langle y^{2}\right\rangle=\lambda_{2}^{2} \\
& R_{4}^{2}=\left\langle\left(z-\frac{\beta_{3}}{\beta_{1}} x\right)^{2}\right\rangle-\left\langle z-\frac{\beta_{3}}{\beta_{1}} x\right\rangle^{2}-\frac{\beta_{3}^{2}}{\beta_{1}^{2}}\left\langle y^{2}\right\rangle \\
& R_{0}^{2}=\left\langle\left(t-\frac{1}{\beta_{1}} x\right)^{2}\right\rangle-\left\langle t-\frac{1}{\beta_{1}} x\right\rangle^{2}-\frac{1}{\beta_{1}^{2}}\left\langle y^{2}\right\rangle .
\end{aligned}
$$

Just as for the correlation radii of (2.15) and (3.3), these fit parameters contain mixtures of various spatial and temporal lengths of homogeneity, making the extraction of source parameters in general highly model dependent. However, a "GYK" fit has the advantage that the time structure of the source enters in only one of the radius parameters, namely $R_{0}^{2}$ 


\section{THE MEASUREMENT FRAME}

In order to illustrate the utility of the saddle-point formalism and the importance of picking the right longitudinal reference frame for making measurements, we will now study three example source models.

\section{A. Static Gaussian Source}

Consider a source function which is defined by spacetime gaussians in its center-of-mass (CM) frame:

$$
S(x, K)=f(K) \exp \left[-\frac{x^{2}+y^{2}}{2 R^{2}}-\frac{z^{2}}{2 L^{2}}-\frac{\left(t-t_{0}\right)^{2}}{2(\Delta t)^{2}}\right] .
$$

Using Eq. (2.2), we see that the saddle point for this function is independent of $\mathbf{K}$ and given by $\bar{x}=\bar{y}=\bar{z}=0$ and $\bar{t}=t_{0}$. In the CM frame, all of the off-diagonal components of $B_{\mu \nu}$ vanish, while the curvature radii are given by

$$
\lambda_{1}=\lambda_{2}=R, \quad \lambda_{3}=L, \quad \text { and } \quad \lambda_{0}=\Delta t .
$$

That the curvature radii are independent of $\mathbf{K}$ is true for any source in which the spacetime and momentum dependences factorize. For the simple source of Eq. (4.1) they simply measure the relevant geometrical "radii" of the system.

If one makes a "standard" fit to the correlation function using CM momentum differences, the extracted radii will have the interpretation

$$
\begin{array}{ll}
R_{1}^{2}=R^{2}+\beta_{1}^{2}(\Delta t)^{2}, & R_{2}^{2}=R^{2} \\
R_{3}^{2}=L^{2}+\beta_{3}^{2}(\Delta t)^{2}, & R_{13}^{2}=\beta_{1} \beta_{3}(\Delta t)^{2}
\end{array}
$$

The duration of emission $(\Delta t)$ can thus be extracted either from $R_{13}^{2}$ or from the difference $R_{1}^{2}-R_{2}^{2}$. If some frame other than the CM is used for making a "standard" fit, then the correlation radii will have much more complicated dependencies on the source parameters. In particular, $R_{1}^{2}-R_{2}^{2}$ will not be proportional to $(\Delta t)^{2}$. 
If a "GYK" fit is made, on the other hand, then in the CM frame or any frame longitudinally boosted from it, one will find the same expressions for the correlation radii:

$$
R_{t}=R, \quad R_{4}=L, \quad R_{0}=\Delta t
$$

Furthermore, the parameter $v$ will measure the velocity difference between the $\mathrm{CM}$ and measurement frames. In other words, even if measurements are made in the "wrong" frame, a "GYK" fit will still produce correlation radii which measure the source in its rest frame.

\section{B. Boost-Invariant Source}

Next we consider a longitudinally expanding, boost-invariant source:

$$
S(x, K)=\frac{m_{t}}{(\Delta \tau)} \exp \left[-\frac{K \cdot u}{T}-\frac{x^{2}+y^{2}}{2 R^{2}}-\frac{\left(\tau-\tau_{0}\right)^{2}}{2(\Delta \tau)^{2}}\right] .
$$

Here $T$ is a constant freeze-out temperature, $\tau=\sqrt{t^{2}-z^{2}}$ is the longitudinal proper time,

and $m_{t}=\sqrt{m^{2}+K_{\perp}^{2}}$. The longitudinal expansion of the source is described by the flow four-velocity relative to some fixed frame

$$
u^{\mu}=(\operatorname{ch} \eta, 0,0, \operatorname{sh} \eta)
$$

where $\eta=\frac{1}{2} \ln [(t+z) /(t-z)]$ is spacetime rapidity.

Calculation of the saddle point in the fixed frame is straightforward, yielding $\bar{x}=\bar{y}=0$, $\bar{\tau}=\tau_{0}$ and $\bar{\eta}=Y$, where $Y$ is the rapidity of a particle with momentum $\mathbf{K}$. Unlike in the previous model, both the saddle point and the curvature tensor depend on $\mathbf{K}$. Explicitly, the curvature radii and off-diagonal elements of $B_{\mu \nu}$ are given by

$$
\begin{aligned}
\lambda_{0} & =\left[\frac{\operatorname{sh}^{2} Y}{\tau_{0}^{2}}\left(\frac{m_{t}}{T}\right)+\frac{\operatorname{ch}^{2} Y}{(\Delta \tau)^{2}}\right]^{-1 / 2} \\
\lambda_{1} & =\lambda_{2}=R \\
\lambda_{3} & =\left[\frac{\operatorname{ch}^{2} Y}{\tau_{0}^{2}}\left(\frac{m_{t}}{T}\right)+\frac{\operatorname{sh}^{2} Y}{(\Delta \tau)^{2}}\right]^{-1 / 2} \\
B_{03} & =B_{30}=-\operatorname{sh} Y \operatorname{ch} Y\left[\frac{1}{\tau_{0}^{2}}\left(\frac{m_{t}}{T}\right)+\frac{1}{(\Delta \tau)^{2}}\right]
\end{aligned}
$$


Here we see that the $z-t$ correlations of the longitudinally expanding source give rise to a nonvanishing $B_{30}$ for pairs with $Y \neq 0$.

The correlation radii from a "standard" fit can be found by plugging these source parameters into Eq. (3.3). One finds

$$
\begin{aligned}
R_{2}^{2} & =R^{2} \\
R_{1}^{2} & =R^{2}+\beta_{1}^{2}\left[(\Delta \tau)^{2} \operatorname{ch}^{2} Y+\left(\frac{T}{m_{t}}\right) \tau_{0}^{2} \operatorname{sh}^{2} Y\right] \\
R_{3}^{2} & =\left(\frac{T}{m_{t}}\right) \frac{\tau_{0}^{2}}{\operatorname{ch}^{2} Y} \\
R_{13}^{2} & =-\beta_{1}\left(\frac{T}{m_{t}}\right) \tau_{0}^{2} \operatorname{th} Y .
\end{aligned}
$$

Using a saddle point approximation to a hydrodynamic model similar to the one presented here, Makhlin and Sinyukov first derived the above expression for $R_{3}^{2}$ [26]. In a recent paper by the NA35 collaboration at CERN, the $m_{t}$ and $Y$ dependence of $R_{3}$ measured in relativistic heavy-ion collisions was compared to that expression in order to estimate a freezeout proper time $\tau_{0}$ [2]. This treatment was not consistent, however, since the parameter $R_{13}^{2}$ was omitted from the fits.

Notice that $R_{1}^{2}-R_{2}^{2}$ in Eqs. (4.8) is in general proportional neither to $(\Delta \tau)^{2}$ nor to $\lambda_{0}^{2}$. This is simply a result of choosing the wrong measurement frame. The extraction of $\Delta \tau$ from correlation radii is greatly simplified if instead of measuring the correlation in a fixed frame, one measures it in the local rest frame of the source near its saddle point $\bar{x}(\mathbf{K})$. At that point, the flow velocity relative to the fixed frame is given by

$$
u=(\operatorname{ch} \bar{\eta}, 0,0, \operatorname{sh} \bar{\eta})
$$

Since $\bar{\eta}=Y$, transforming to the local rest frame (primed) of the saddle point can be done for each pair by making the following substitutions

$$
\begin{gathered}
\eta^{\prime}=\eta-\bar{\eta}=\eta-Y, \quad Y^{\prime}=Y-\bar{\eta}=0 \\
q_{3}^{\prime}=\operatorname{ch} Y\left(q_{3}-\operatorname{th} Y q_{0}\right), \quad q_{0}^{\prime}=\operatorname{ch} Y\left(q_{0}-\operatorname{th} Y q_{3}\right) .
\end{gathered}
$$


In other words, the local rest frame of a boost-invariant source is just the LCMS $\left(Y^{\prime}=0\right.$ frame).

It is easy to verify that in this frame $B_{30}^{\prime}$ vanishes and

$$
\lambda_{0}^{\prime}=\Delta \tau, \quad \lambda_{1}^{\prime}=\lambda_{2}^{\prime}=R, \quad \lambda_{3}^{\prime}=\tau_{0} \sqrt{T / m_{t}} .
$$

Due to the diagonal nature of the curvature tensor, these source parameters can be easily extracted from the LCMS correlation radii

$$
R_{1}^{\prime 2}=R^{2}+\beta_{1}^{2}(\Delta \tau)^{2}, \quad R_{2}^{\prime 2}=R^{2}, \quad R_{3}^{\prime 2}=\left(T / m_{t}\right) \tau_{0}^{2}, \quad R_{13}^{\prime 2}=0 .
$$

Note that in this frame $R_{1}^{2}-R_{2}^{2}$ is proportional to $(\Delta \tau)^{2}$. Using the "standard" fitting procedure, it obviously makes the most sense to measure correlations for boost-invariant sources in the LCMS rather than a fixed frame.

Using the "GYK" fitting procedure, on the other hand, one finds that regardless of the longitudinal frame chosen,

$$
R_{t}=R, \quad R_{4}=\tau_{0} \sqrt{T / m_{t}}, \quad R_{0}=\Delta \tau,
$$

while $v$ measures the difference between the measurement frame and the local rest frame of the source at the saddle point. For example, a measurement made in the LCMS would yield $v=0$, while one made in a fixed frame would yield $v=\operatorname{th} Y$ for pairs with an average rapidity $Y$ relative to that frame.

\section{Finite Expanding Source}

The main problem with a boost-invariant source is that it gives rise to a $d N / d y$ which is completely flat, whereas the $d N / d y$ for produced particles which are actually observed in relativistic heavy-ion collisions are much better described by gaussians in rapidity. Furthermore, in a boost-invariant source, $R_{13}^{\prime 2}$ will vanish in the LCMS, but nonzero values for $R_{13}^{\prime 2}$ have been measured by NA35 [27]. These inconsistencies with boost-invariant sources lead us to consider an expanding model with a non-boost-invariant cutoff in spacetime rapidity 
[8.14,28,29]. Using the expansion four-velocity of Eq. (4.6), we define a source in its CM frame by

$$
S(x, K)=\frac{m_{t} \operatorname{ch}(\eta-Y)}{(2 \pi)^{3} \sqrt{2 \pi(\Delta \tau)^{2}}} \exp \left[-\frac{K \cdot u}{T}-\frac{x^{2}+y^{2}}{2 R^{2}}-\frac{\left(\tau-\tau_{0}\right)^{2}}{2(\Delta \tau)^{2}}-\frac{\eta^{2}}{2(\Delta \eta)^{2}}\right] .
$$

In [8] it was shown that the $\Delta \eta$ cutoff term leads to more realistic gaussian-like rapidity distributions. The prefactor was introduced so that in the limit as $\Delta \tau \rightarrow 0, S(x, K)$ becomes the Boltzmann approximation to a hydrodynamical source which freezes out at a constant temperature $T$ and proper time $\tau_{0}$ [30.

The saddle point for this model still has the coordinates $\bar{x}=\bar{y}=0$ and $\bar{\tau}=\tau_{0}$, but the spacetime-rapidity coordinate for a given value of $\mathbf{K}$ (relative to the $\mathrm{CM}$ frame) is now given by the solution of

$$
\operatorname{th}(\bar{\eta}-Y)-\frac{m_{t}}{T} \operatorname{sh}(\bar{\eta}-Y)-\frac{\bar{\eta}}{(\Delta \eta)^{2}}=0 .
$$

It is apparent that the saddle point is only located at $\bar{\eta}=Y$ for an infinite longitudinal tube $\Delta \eta \rightarrow \infty$ or for pairs with $Y=0$.

As in the previous model, transforming to the local rest frame (primed) at the saddle point can be achieved by

$$
\begin{gathered}
\eta^{\prime}=\eta-\bar{\eta}, \quad Y^{\prime}=Y-\bar{\eta} \\
q_{3}^{\prime}=\operatorname{ch} \bar{\eta}\left(q_{3}-\operatorname{th} \bar{\eta} q_{0}\right), \quad q_{0}^{\prime}=\operatorname{ch} \bar{\eta}\left(q_{0}-\operatorname{th} \bar{\eta} q_{3}\right) .
\end{gathered}
$$

Notice that since $Y^{\prime} \neq 0$, the local rest frame does not coincide with the LCMS. It is possible to show that in the local rest frame the curvature tensor becomes diagonal $\left(B_{30}^{\prime}=0\right)$ and

$$
\begin{gathered}
\lambda_{1}^{\prime}=\lambda_{2}^{\prime}=R, \quad \lambda_{0}^{\prime}=\Delta \tau, \\
\lambda_{3}^{\prime}=\tau_{0}\left[\frac{m_{t}}{T} \operatorname{ch}(\bar{\eta}-Y)-\frac{1}{\operatorname{ch}^{2}(\bar{\eta}-Y)}+\frac{1}{(\Delta \eta)^{2}}\right]^{-1 / 2} .
\end{gathered}
$$

In the CM and LCMS frames, however, the curvature tensor is in general not diagonal and each component becomes much more complicated. These complications carry over into the 
correlation radii. For example, $R_{13}^{2}$ does not vanish in these frames, and $R_{1}^{2}-R_{2}^{2}$ is not proportional to either $(\Delta \tau)^{2}$ or $\lambda_{0}^{2}$, 8,14 .

By looking at one-particle slopes and rapidity distributions, it may be possible to estimate $T$ and $\Delta \eta$. Using these values, one can numerically solve Eq. (4.15) for $\bar{\eta}(\mathbf{K})$, use Eq. (4.16)

to transform to the local rest frame, and then extract the source parameters by making a "standard" fit to the correlation function. Alternatively, one could simply make a "GYK" fit in the CM frame (or any other longitudinally boosted frame), and the result would be

$$
R_{t}=\lambda_{1}^{\prime}=\lambda_{2}^{\prime}, \quad R_{4}=\lambda_{3}^{\prime}, \quad R_{0}=\lambda_{0}^{\prime}
$$

Again the parameter $v(\mathbf{K})$ would measure the velocity difference between the measurement frame and the local rest frame.

Each of the three models discussed above exhibits a different local rest frame. If one makes a "standard" fit to the correlation function, it is important to guess the correct reference frame before performing the fit in order to extract useful information about the source. This implies that one must have some a priori knowledge about the source before making the fit. If one makes a "GYK" fit, however, there is no need to pick a frame beforehand. In all three of the above cases, the fitting procedure itself automatically chooses the correct frame in which to measure the source. This feature of the "GYK" fitting procedure is actually common to a whole class of models we will discuss below.

\section{A Class of Models}

All of the models we have discussed so far belong to a class of source functions which satisfy the following conditions:

$$
\lambda_{1}=\lambda_{2} \quad \text { and } \quad B_{10}=B_{31}=0
$$

For models in this class, the curvature tensor (2.4) takes the simple block-diagonal form 


$$
B_{\mu \nu}=\left(\begin{array}{cccc}
\lambda_{2}^{-2} & 0 & 0 & 0 \\
0 & \lambda_{2}^{-2} & 0 & 0 \\
0 & 0 & \lambda_{3}^{-2} & B_{30} \\
0 & 0 & B_{30} & \lambda_{0}^{-2}
\end{array}\right) .
$$

Moreover its inverse, the correlation matrix $B^{-1}$ of Eqs. (2.11) and (2.9), has the same block-diagonal structure, i.e., the $x-t$ and $x-z$ correlations $\left(B^{-1}\right)_{10}$ and $\left(B^{-1}\right)_{13}$ vanish, and $\left(B^{-1}\right)_{11}=\left(B^{-1}\right)_{22}$.

Since models in this class have only four non-vanishing components of the curvature tensor, these four can be unambiguously determined by measuring the four parameters coming from either a "standard" or "GYK" fit. Explicitly,

$$
\begin{aligned}
& R_{2}=\lambda_{2}=R_{t} \\
& R_{3}^{2}-\frac{\left(R_{13}^{2}\right)^{2}}{\left(R_{1}^{2}-R_{2}^{2}\right)}=\lambda_{3}^{2}=\frac{R_{0}^{2} R_{4}^{2}}{\gamma^{2}\left(R_{0}^{2}+v^{2} R_{4}^{2}\right)} \\
& \frac{\left(R_{1}^{2}-R_{2}^{2}\right) R_{3}^{2}-\left(R_{13}^{2}\right)^{2}}{\beta_{1}^{2} R_{3}^{2}-2 \beta_{1} \beta_{3} R_{13}^{2}+\beta_{3}^{2}\left(R_{1}^{2}-R_{2}^{2}\right)}=\lambda_{0}^{2}=\frac{R_{0}^{2} R_{4}^{2}}{\gamma^{2}\left(R_{4}^{2}+v^{2} R_{0}^{2}\right)} \\
& \frac{\beta_{1} R_{13}^{2}-\beta_{3}\left(R_{1}^{2}-R_{2}^{2}\right)}{\left(R_{1}^{2}-R_{2}^{2}\right) R_{3}^{2}-\left(R_{13}^{2}\right)^{2}}=B_{30}=-\frac{v \gamma^{2}\left(R_{0}^{2}+R_{4}^{2}\right)}{R_{0}^{2} R_{4}^{2}},
\end{aligned}
$$

where $\gamma$ is defined in (3.8). If the $q$ used for fitting the correlation function are evaluated in a fixed frame, then the above parameters describe the source as seen in that fixed frame. If, on the other hand, LCMS values for $q_{i}$ are used, then Eqs. (4.21) (with $\beta_{3}=0$ ) will determine LCMS source parameters.

An interesting experimental test is provided by comparing the left and right sides of Eqs. (4.21). Namely, if after making both "standard" and "GYK" fits to the correlation function it is found that the left and right sides of Eqs. (4.21) are not equal, then the source in question cannot belong to the class (4.19).

As we mentioned previously, the form of the "GYK" fit means that the extracted parameters $R_{t}(\mathbf{K}), R_{4}(\mathbf{K})$, and $R_{0}(\mathbf{K})$ naturally measure the source in the $v(\mathbf{K})=0$ frame. Setting $v=0$ in Eqs. (4.21), we see that the right halves of the first three equations reduce to Eqs. (4.18). In other words, the $R$ parameters directly measure the curvature radii of 
the source in the $v(\mathbf{K})=0$ frame. Furthermore, the last equation in (4.21) shows us that the $v(\mathbf{K})=0$ frame corresponds to the frame in which $B_{30}(\mathbf{K})$ vanishes $(z-t$ correlations vanish) and the curvature tensor diagonalizes. For many interesting models including the three discussed above, this frame also corresponds to the local rest frame of the source at the saddle point $\bar{x}(\mathbf{K})$.

As for the "standard" fitting procedure, the left halves of Eqs. (4.21) tell us that only if we are clever enough to pick the $B_{30}=0$ frame beforehand will $\lambda_{0}^{2}$ reduce to the more familiar form

$$
\lambda_{0}^{2}=\frac{1}{\beta_{1}^{2}}\left(R_{1}^{2}-R_{2}^{2}\right)
$$

Using RQMD events in the LCMS, this equation was recently shown to be a good approximation only for pairs with very small $Y$ and $K_{\perp}$ in the center-of-mass frame of a symmetric projectile-target collision [19]. The restriction of small $Y$ found by these authors can be explained in the following way: Only at $Y=0$ do the LCMS and the local rest frame definitely coincide for all sources resulting from a symmetric projectile-target collision. As $|Y|$ increases, these two frames may begin to diverge just as they did for the model discussed

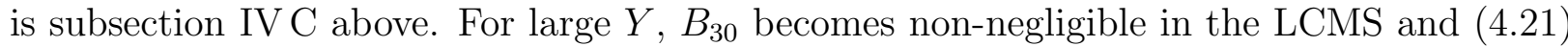
rather than (4.22) must be used to extract $\lambda_{0}$. As we will show in the next section, the additional restriction of small $K_{\perp}$ can be explained if a source is undergoing transverse as well as longitudinal expansion.

The reader should note that there is a difficulty with the "standard" fitting procedure which arises due to the fact that experimental correlation functions are always generated for pairs which lie in certain bins in $K_{\perp}$ and $K_{3}=K_{L}$ rather than for exact values of these average momenta. In extracting $\lambda_{0}$ and $B_{30}$ of Eqs. (4.21) from the left-hand expressions, there will be a certain ambiguity as to which values of $\beta_{1}$ and $\beta_{3}$ should be used. Fortunately, this problem can be circumvented by employing the new fitting procedures that we introduce in Appendix A. Since no $\beta_{i}$ are present on the right side of Eqs. (4.21), the difficulty does not arise at all when one uses the "GYK" fitting procedure. 
Using the generalized Yano-Koonin formalism has the further advantage that by making a single fit in a fixed frame, one can determine the source parameters both in that frame via (4.21) and simultaneously in the $v(\mathbf{K})=0$ frame via (4.18). Similarly, by making a single fit in the LCMS frame, one can simultaneously determine both LCMS and $v(\mathbf{K})=0$ source parameters. Using the "standard" fitting procedure, on the other hand, requires at least two fits (see Appendix A) just to determine the source parameters in a single frame.

Finally, we would like to discuss the reason why we chose to study the class of models defined by Eqs. (4.19). First of all, as we have seen explicitly, there are a number of interesting models which naturally fall into this class. More importantly, however, the source function for pairs with $K_{\perp}=0$ from any azimuthally symmetric model will always fall into this class. The reason for this is simply because when $K_{\perp}=0$, there is no way to distinguish between the "side" and "out" directions. Consequently, $\lambda_{1}=\lambda_{2}$ and since $B_{2 \mu}=0$, it must also be true that $B_{1 \mu}=0$. If all models fall into this class for pairs with $K_{\perp}$ exactly vanishing, then there should be a wide range of models which are "close" to being in this class for pairs with sufficiently small $K_{\perp}$. In the next section, we will look at an illustrative example model.

\section{A MODEL WITH TRANSVERSE EXPANSION}

Just as longitudinally expanding sources feature $z$-t correlations in their CM frame, transversally expanding sources will in general feature $x$-t correlations in their CM frame or in any frame which is only longitudinally boosted relative to it. Consequently, such sources will exhibit nonvanishing $B_{10}$ and/or $B_{31}$ and thus not belong to the class (4.19). Nevertheless, by working with Eqs. (2.5) as well as the corresponding general expressions

for the "GYK" fit, it can be shown that if for some range of $\mathbf{K}$, all of the $\lambda_{\mu}$ are of the same order and

$$
\left(\lambda_{1} \lambda_{0} B_{10}\right)^{2} \ll \beta_{1}^{2}, \quad\left(\lambda_{3} \lambda_{1} B_{31}\right)^{2} \ll \beta_{1}^{2}, \quad \text { and } \quad 1-\lambda_{2}^{2} / \lambda_{1}^{2} \ll \beta_{1}^{2},
$$


then Eqs. (4.21) are still good approximations. In other words, Eqs. (5.1) define a larger class of models for which it is possible to unambiguously extract the parameters $\lambda_{1}(\mathbf{K}) \simeq \lambda_{2}(\mathbf{K})$, $\lambda_{3}(\mathbf{K}), \lambda_{0}(\mathbf{K})$, and $B_{30}(\mathbf{K})$. Furthermore, from Eq. (3.9) it can be verified that if Eqs. (5.1) hold, then in the $v(\mathbf{K})=0$ frame, $\lambda_{0}^{2} \lambda_{3}^{2} B_{30}^{2} \ll 1$, so it is a good approximation to treat the curvature tensor as being diagonal, and the correlation radii in this frame are given by Eqs. (4.18).

We will now examine a specific source model which exhibits transverse as well as longitudinal expansion to see what kind of restrictions Eqs. (5.1) impose on the average momentum K. We consider a source function of the form (4.14), but with an expansion four-velocity now given by

$$
u(x)=\left(\sqrt{1+\left(v_{t} \rho / R\right)^{2}} \operatorname{ch} \eta,\left(v_{t} x / R\right),\left(v_{t} y / R\right), \sqrt{1+\left(v_{t} \rho / R\right)^{2}} \operatorname{sh} \eta\right) .
$$

Since $u(x)$ is well defined and $u \cdot u=1$ for arbitrarily large $v_{t}$, Eq. (5.2) can be used for modeling relativistic $\left(v_{t} \gtrsim 1\right)$ as well as nonrelativistic $\left(v_{t} \ll 1\right)$ transverse expansions. (Only for nonrelativistic expansions does the parameter $v_{t}$ represent the transverse velocity of the source at $\rho=R$.)

By using Eq. (2.2) to calculate the saddle point of the emission function and recalling that $\mathbf{K}$ does not have any component in the $\hat{y}$ ("side") direction, it is easily found that $\bar{\tau}=\tau_{0}$ and $\bar{y}=0$. Similarly, one can show that

$$
\frac{v_{t} \bar{x}}{R}=\frac{K_{\perp} v_{t}^{2} / T}{1+\left(m_{t} v_{t}^{2} / T\right) \operatorname{ch}(\bar{\eta}-Y)\left[1+\left(v_{t} \bar{x} / R\right)^{2}\right]^{-1 / 2}}
$$

and

$$
1-\frac{\lambda_{2}^{2}}{\lambda_{1}^{2}}=\frac{\left(v_{t} \bar{x} / R\right)^{2}\left(m_{t} v_{t}^{2} / T\right) \operatorname{ch}(\bar{\eta}-Y)}{\left(m_{t} v_{t}^{2} / T\right) \operatorname{ch}(\bar{\eta}-Y)\left[1+\left(v_{t} \bar{x} / R\right)^{2}\right]+\left[1+\left(v_{t} \bar{x} / R\right)^{2}\right]^{3 / 2}} .
$$

From these equations, it can be seen that if we demand that

$$
E_{K}<\frac{T}{v_{t}^{2}},
$$

then $1-\lambda_{2}^{2} / \lambda_{1}^{2}$ will always be less than $\beta_{1}^{2}$. Actually, given the form of Eqs. (5.3) and (5.4), we are justified in deducing that Eq. (5.5) implies $1-\lambda_{2}^{2} / \lambda_{1}^{2} \ll \beta_{1}^{2}$ rather than the 
weaker condition $1-\lambda_{2}^{2} / \lambda_{1}^{2}<\beta_{1}^{2}$. In Appendix B we also show that if (5.5) is satisfied, then $\left(\lambda_{1} \lambda_{0} B_{10}\right)^{2} \ll \beta_{1}^{2}$ and $\left(\lambda_{3} \lambda_{1} B_{31}\right)^{2} \ll \beta_{1}^{2}$. Consequently, in the context of the present model, Eq. (5.5) is a sufficient condition to justify the use of the expressions in the last section.

In particular, if one makes a "GYK" fit to the correlation function of the model under consideration, then for pairs satisfying (5.5), the extracted correlation radii will measure the source in the local longitudinal rest frame at the saddle point. Explicitly,

$$
\begin{gathered}
R_{0} \simeq \lambda_{0} \simeq \Delta \tau, \quad R_{t} \simeq \lambda_{1} \simeq \lambda_{2} \simeq R\left[1+\frac{m_{t} v_{t}^{2}}{T} \operatorname{ch}(\bar{\eta}-Y)\right]^{-1 / 2} \\
R_{4} \simeq \lambda_{3} \simeq \tau_{0}\left[\frac{m_{t}}{T} \operatorname{ch}(\bar{\eta}-Y)-\frac{1}{\operatorname{ch}^{2}(\bar{\eta}-Y)}+\frac{1}{(\Delta \eta)^{2}}\right]^{-1 / 2}
\end{gathered}
$$

where $\bar{\eta}(\mathbf{K}) \simeq \operatorname{th}^{-1}[v(\mathbf{K})]$. By looking at the $m_{t}$-dependence of $R_{t}$, it may be possible to extract both the transverse size $R$ and the expansion parameter $v_{t}^{2} / T$. Using the latter, it is possible to make a consistency check to see if the pairs under consideration did in fact satisfy condition (5.5).

The temperature $T$ and transverse velocity parameter $v_{t}$ can also be determined by measuring slopes and curvatures of one-particle distributions. In heavy-ion collisions at the

both the AGS and SPS, these parameters have been estimated to be on the order of $T=140$ $\mathrm{MeV}$ and $v_{t}=0.5$ [31 33. From Eq. (5.5), these estimates imply that Eqs. (5.6) should be good approximations for pairs with $E_{K}$ less than about $560 \mathrm{MeV}$.

\section{CONCLUSIONS}

We have shown that in general the number of parameters needed to describe a source in the quadratic saddle-point approximation exceeds the number of parameters which can be determined by making gaussian fits to two-particle correlation data. However, we have identified a wide class of interesting models for which the source is fully described by only K-dependent parameters which can all be determined from the experimental parameters measured in a gaussian fit. Using a realistic three-dimensionally expanding hydrodynamical model, we showed that for heavy-ion collisions at the AGS or SPS, it should be simplest 
to extract the source parameters from correlations of pairs with average energies less than about $560 \mathrm{MeV}$ in the measurement frame. It should be noted that the source shape seen by these particles may still not be the geometrical shape of the source, but rather that of the local region of homogeneity which is affected by the expansion flow profile of the source. To separate the flow effect from the underlying geometry, it is necessary to determine the $K_{\perp}$ dependence of the HBT radius parameters. Fortunately, much of this dependence may be possible to see while staying in the "simple" regime. For example, for pions with $K_{L}=0$ in the measurement frame, values of $K_{\perp}$ up to $540 \mathrm{MeV} / \mathrm{c}$ will still correspond to energies below $560 \mathrm{MeV}$, so at least five $100-\mathrm{MeV}$ bins in $K_{\perp}$ can be explored below the limit. Of course for any given analytic model it should also be possible to extract source parameters from large $K_{\perp}$ correlation radii by using expressions such as those in Eqs. (3.3). However, at large $K_{\perp}$, each correlation radius will contain contributions from a large number of effects which may be difficult to disentangle.

In the past there has been some debate as to which longitudinal reference frame would be the most appropriate for measuring correlations from a given reaction. For example, for a source which is not expanding longitudinally, the source center-of-mass frame is the natural choice. On the other hand, for an infinite source which is undergoing a boost-invariant expansion, the LCMS represents the local rest frame of the source and is thus the natural choice. Since experimental reactions undoubtedly produce sources which lie somewhere between these two extremes, some intermediate frame is needed. The generalized YanoKoonin fitting procedure of Eq. (3.7) has the advantage that it does not require one to postulate a reference frame beforehand; the data themselves determine a frame for each value of $\mathbf{K}$. For many interesting "intermediate" models, the parameters in this $v(\mathbf{K})=0$ frame measure the source in its local longitudinal rest frame, while fixed frame or LCMS parameters measure the source in some different frame.

Certainly the best way to compare any given model to correlation data is to make the comparison directly in a six-dimensional $\left(\mathbf{p}_{1}, \mathbf{p}_{2}\right)$ space, rather than to compare the fitted correlation radii of the model to those extracted from the data. Nevertheless, we have shown 
here that gaussian fits can still reveal some very interesting information about the velocity of the local longitudinal rest frame as well as the lengths of homogeneity of the source.

\section{ACKNOWLEDGMENTS}

The authors would like to thank Pierre Scotto, Urs Wiedemann, Doug Fields and Nu

$\mathrm{Xu}$ for fruitful discussions and constructive comments. The work of U.H. was supported by the Deutsche Forschungsgemeinschaft (DFG), Gesellschaft für Schwerionenforschung (GSI) and Bundesministerium für Bildung und Forschung (BMBF). The work of S.C. and J.R.N. was supported by the US Department of Energy.

\section{APPENDIX A:}

In this appendix we show how the $\beta_{i}$ dependencies in $\lambda_{0}$ and $B_{30}$ of Eqs. (4.21) can be removed through the introduction of new fitting procedures. We label the fitting procedure defined by Eq. (3.2) with an " $a$ ". Thus, $R_{3}^{2}(a)$ refers to the square of the "longitudinal" radius as found by fitting the correlation with Eq. (3.2). We define a "b" fitting procedure by using $\beta_{1} q_{3}$ instead of $q_{3}$ in making a gaussian fit to the correlation function. In other words,

$$
C(\mathbf{q}, \mathbf{K})=1 \pm \lambda \exp \left[-q_{1}^{2} R_{1}^{2}(b)-q_{2}^{2} R_{2}^{2}(b)-\left(\beta_{1} q_{3}\right)^{2} R_{3}^{2}(b)-2 q_{1}\left(\beta_{1} q_{3}\right) R_{13}^{2}(b)\right]
$$

where we have suppressed the $\mathbf{K}$ dependence of the $R^{2}$ parameters. It can now be seen that in the LCMS (primed) frame

$$
\begin{aligned}
\lambda_{0}^{\prime 2} & =\frac{\left[R_{1}^{\prime 2}(a)-R_{2}^{\prime 2}(a)\right] R_{3}^{\prime 2}(a)-\left[R_{13}^{\prime 2}(a)\right]^{2}}{R_{3}^{\prime 2}(b)} \\
B_{30}^{\prime} & =\frac{R_{13}^{\prime 2}(b)}{\left[R_{1}^{\prime 2}(a)-R_{2}^{\prime 2}(a)\right] R_{3}^{\prime 2}(a)-\left[R_{13}^{\prime 2}(a)\right]^{2}} .
\end{aligned}
$$

To calculate $\lambda_{0}$ and $B_{30}$ in a fixed frame, we need to introduce two additional fitting procedures. Procedure " $c$ " is defined by using $\beta_{3} q_{1}, \beta_{3} q_{2}$, and $\beta_{1} q_{3}$ in place of $q_{1}, q_{2}$, and 
$q_{3}$ when making gaussian fits, while procedure " $d$ " is defined by using $\sqrt{\left|\beta_{3}\right|} q_{1}, \sqrt{\left|\beta_{3}\right|} q_{2}$, and $q_{3}$. We then have

$$
\begin{aligned}
\lambda_{0}^{2} & =\frac{\left[R_{1}^{2}(a)-R_{2}^{2}(a)\right] R_{3}^{2}(a)-\left[R_{13}^{2}(a)\right]^{2}}{R_{3}^{2}(c)-2 R_{13}^{2}(c)+R_{1}^{2}(c)-R_{2}^{2}(c)} \\
B_{30} & =\frac{R_{13}^{2}(b) \mp\left[R_{1}^{2}(d)-R_{2}^{2}(d)\right]}{\left[R_{1}^{2}(a)-R_{2}^{2}(a)\right] R_{3}^{2}(a)-\left[R_{13}^{2}(a)\right]^{2}},
\end{aligned}
$$

where the $-(+)$ sign in $B_{30}$ refers to bins in which $\beta_{3}>0\left(\beta_{3}<0\right)$. Note that due to this distinction, the above method for determining source parameters should not be applied to bins in which some of the pairs have $\beta_{3}>0$ while others have $\beta_{3}<0$.

\section{APPENDIX B:}

To prove the remaining inequalities in Eq. (5.1), we begin by presenting the following easily verified relations:

$$
\begin{aligned}
B_{31} & =\frac{m_{t} v_{t}}{R T}\left(\frac{v_{t} \bar{x}}{R}\right)\left(\frac{\bar{t}}{\tau_{0}^{2}}\right) \frac{\operatorname{sh}(\bar{\eta}-Y)}{\sqrt{1+\left(v_{t} \bar{x} / R\right)^{2}}} \\
B_{10} & =-\frac{m_{t} v_{t}}{R T}\left(\frac{v_{t} \bar{x}}{R}\right)\left(\frac{\bar{z}}{\tau_{0}^{2}}\right) \frac{\operatorname{sh}(\bar{\eta}-Y)}{\sqrt{1+\left(v_{t} \bar{x} / R\right)^{2}}} \\
\frac{1}{\lambda_{1}^{2}} & >\frac{m_{t} v_{t}^{2}}{R^{2} T} \frac{\operatorname{ch}(\bar{\eta}-Y)}{\left[1+\left(v_{t} \bar{x} / R\right)^{2}\right]^{3 / 2}} .
\end{aligned}
$$

The saddle point in spacetime rapidity for this source is given by

$$
\operatorname{th}(\bar{\eta}-Y)-\frac{m_{t}}{T} \sqrt{1+\left(v_{t} \bar{x} / R\right)^{2}} \operatorname{sh}(\bar{\eta}-Y)-\frac{\bar{\eta}}{(\delta \eta)^{2}}=0
$$

To derive the needed expressions for $\lambda_{0}^{2}$ and $\lambda_{3}^{2}$, we have first used Eq. (B2) to check numerically that if $\left(m_{t} / T\right) \sqrt{1+\left(v_{t} \bar{x} / R\right)^{2}}>0.7$, then for $Y \neq 0,|\bar{\eta}|<|Y|$ and $\bar{\eta}$ has the same sign as $Y$. Although $\left(m_{t} / T\right) \sqrt{1+\left(v_{t} \bar{x} / R\right)^{2}}>0.7$ may present a significant restriction for electron or photon correlation measurements, it does not present a significant restriction for current two-hadron correlation measurements from particle or heavy-ion collisions. Even for pions, temperatures of up to $200 \mathrm{MeV}$ would still satisfy this condition. Given this condition, from Eq. (B2) it is possible to show that for $Y \neq 0$, 


$$
\frac{1}{\operatorname{ch}(\bar{\eta}-Y)}<\frac{m_{t}}{T} \sqrt{1+\left(v_{t} \bar{x} / R\right)^{2}}
$$

Using this inequality, then for all $Y$ it is true that

$$
\begin{aligned}
& \lambda_{0}^{-2}>\frac{m_{t}}{T} \sqrt{1+\left(v_{t} \bar{x} / R\right)^{2}}\left(\frac{\bar{z}}{\tau_{0}^{2}}\right)^{2} \frac{\operatorname{sh}^{2}(\bar{\eta}-Y)}{\operatorname{ch}(\bar{\eta}-Y)} \\
& \lambda_{3}^{-2}>\frac{m_{t}}{T} \sqrt{1+\left(v_{t} \bar{x} / R\right)^{2}}\left(\frac{\bar{t}}{\tau_{0}^{2}}\right)^{2} \frac{\operatorname{sh}^{2}(\bar{\eta}-Y)}{\operatorname{ch}(\bar{\eta}-Y)} .
\end{aligned}
$$

The desired inequalities are now easily proven:

$$
\begin{aligned}
& \left(\lambda_{1} \lambda_{3} B_{31}\right)^{2}<\left(v_{t} \bar{x} / R\right)^{2} \ll \beta_{1}^{2} \\
& \left(\lambda_{1} \lambda_{0} B_{10}\right)^{2}<\left(v_{t} \bar{x} / R\right)^{2} \ll \beta_{1}^{2} .
\end{aligned}
$$




\section{REFERENCES}

[1] D. Boal, C.K. Gelbke, and B. Jennings, Rev. Mod. Phys. 62, 553 (1990).

[2] NA35 Coll., T. Alber et al., Z. Phys. C66, 77 (1995).

[3] NA35 Coll., G. Roland et al., Nucl. Phys. A566, 527c (1994); NA35 Coll., T. Alber et al., Phys. Rev. Lett. 74, 1303 (1995); NA44 Coll., M. Sarabura et al., Nucl. Phys. A544, 125c (1992); E802 Coll., T. Abbott et al., Phys. Rev. Lett. 69, 1030 (1992).

[4] NA35 Coll., P. Seyboth et al., Nucl. Phys. A544, 293c (1992); NA35 Coll., D. Ferenc et al., Nucl. Phys. A544, 531c (1992).

[5] NA44 Coll., H. Beker et al., Phys. Rev. Lett. 74, 3340 (1995).

[6] Yu.M. Sinyukov, in "Hot Hadronic Matter: Theory and Experiment," edited by J. Letessier et al. (Plenum, New York, 1995).

[7] S.V. Akkelin and Yu.M. Sinyukov, Bogolyubov preprint ITP-63-94E, Phys. Lett. B, in press.

[8] S. Chapman, P. Scotto, and U. Heinz, Los Alamos e-print archive hep-ph/9409349, Heavy Ion Physics 1, 1 (1995).

[9] F. Yano and S. Koonin, Phys. Lett. B78, 556 (1978).

[10] W. Zajc, Nucl. Phys. A525, 315c (1991).

[11] S. Pratt, T. Csörgő, and J. Zimányi, Phys. Rev. C42, 2646 (1990).

[12] S. Chapman and U. Heinz, Phys. Lett. B340, 250 (1994).

[13] G. Bertsch, P. Danielewicz, and M. Herrmann, Phys. Rev. C49, 442 (1994); M. Herrmann and G. Bertsch, Phys. Rev. C51, 328 (1995).

[14] S. Chapman, P. Scotto, and U. Heinz, Phys. Rev. Lett. 74, 4400 (1995).

[15] M. Gyulassy, S.K. Kauffmann, and L.W. Wilson, Phys. Rev. C20, 2267 (1979). 
[16] R.M. Weiner pointed out in Phys. Lett. B232, 278 (1989) that in the case that the source is partially coherent the correlation function should in fact not be fitted to a single gaussian, but to a sum of gaussians with radius parameters differing by a factor $\sqrt{2}$.

[17] J. Bolz, U. Ornik, M. Plumer, B. Schlei, and R. Weiner, Phys. Lett. B300, 404 (1993).

[18] T. Csörgö, B. Lørstad, and J. Zimányi, Lund preprint LUNFD6-NFFL-7088-1994, Phys. Lett. B, submitted.

[19] D. Fields, J. Sullivan, B. Jacak, N. Xu, J. Simon-Gillo, and H. van Hecke, Phys. Rev. C, submitted.

[20] J. Sullivan, M. Berenguer, B. Jacak, M. Sarabura, J. Simon-Gillo, H. Sorge, H. van Hecke, and S. Pratt, Phys. Rev. Lett. 70, 3000 (1993).

[21] T. Csörgö and S. Pratt, Lund preprint LU TP 91-10, in: Proc. of the Workshop on Relativistic Heavy Ion Physics, preprint KFKI-1991-28/A, p. 75.

[22] U.A. Wiedemann, P. Scotto, and U. Heinz, Transverse momentum dependence of HBT correlation radii, Regensburg preprint TPR-95-12 (Aug. 1995), unpublished.

[23] G. Bertsch, M. Gong, and M. Tohyama, Phys. Rev. C37, 1896 (1988).

[24] S. Chapman, P. Scotto, and U. Heinz, Talk given at Quark Matter '95 in Monterey, January 9-13, 1995, Nucl. Phys. A, to be published.

[25] Several such fits are made and the phase-space limitations discussed in the 1994 University of Michigan Ph.D. thesis of Vince Cianciolo.

[26] A.M. Makhlin and Yu.M. Sinyukov, Z. Phys. C39, 69 (1988). Yu.M. Sinyukov, Nucl. Phys. A498, 151c (1989).

[27] NA35 Coll., Th. Alber, private communication. 
[28] T. Csörgő, Phys. Lett. B347, 354 (1995).

[29] S. Padula and M. Gyulassy, Nucl. Phys. A498, 555c (1988).

[30] B.R. Schlei, U. Ornik, M. Plümer, and R.M. Weiner, Phys. Lett. B293, 275 (1992).

[31] E. Schnedermann and U. Heinz, Phys. Rev. Lett. 69, 2908 (1992); E. Schnedermann, J. Sollfrank, and U. Heinz, Phys. Rev. C48, 2462 (1993); E. Schnedermann and U. Heinz, Phys. Rev. C50, 1675 (1994).

[32] P. Braun-Munzinger, J. Stachel, J. Wessels, and N. Xu, Phys. Lett. B344, 43 (1995).

[33] B. Jacak, Talk given at Quark Matter '95 in Monterey, January 9-13, 1995, Nucl. Phys. A, to be published. 